\title{
Interference and Noise detection in a Cognitive Radio Environment
}

\author{
Pradeep. $\mathrm{R}^{1}$, Gopinath. $\mathrm{T}^{2}$ \\ I'Computer Science, Anna University, India) \\ ${ }^{2}$ (Electronics and communication, Anna University, India)
}

\begin{abstract}
Nowadays cellular and handheld devices hold up most of the data exchange services that is one currently and they widely use high speed data links causing increased usage of available spectrum. However these devices are not always in use so the links stays void sometime and using those void links in spectrum is the brilliance of cognitive radio. When the primary user doesn't avail his licensed band, secondary users use those bands using cognitive radio technology so that the bands are put to efficient usage all the time. Since artificial intelligence is responsible for that we are allowing unanticipated, emergent behavior of the sensors in the radio by which an adversary can easily manipulate the radio and could change its behavior. In this paper we primarily focus on one such adversaries effect and how to overcome it.
\end{abstract}

Keywords: Cognitive Radio, interference, spectrum

\section{INTRODUCTION}

Cognitive radio offers the advantage of intelligent radios which have the ability to sense free band and make it useable for secondary users. Due to this behavior of those they are easily prone to hackers and external adversaries to walk them from a path of right sensing. The sensors used in cognitive radio uses artificial intelligence to sense whether a band is in use or not. If the hacker can produce a signal similar to those of the signal the private users use then the sensor will think that the primary user is availing the band and hence the sensor moves away from that band and starts sensing for other bands.

Also if the primary users occupy the band and there is lot of noise generated from the environment the sensor does the same action of moving away and sensing for other bands. If the sensor could differentiate whether the occupied signal is either noise or interference signal then the sensor can either decide to use that band or not depending on the result. In this paper we will study about how to differentiate between an interference and a noise signal. There are two sections. Section 1 to give a detailed analyses on differentiation and section 2 is conclusion.

\section{DETAILED ANALYSES}

\section{Prototype model:}

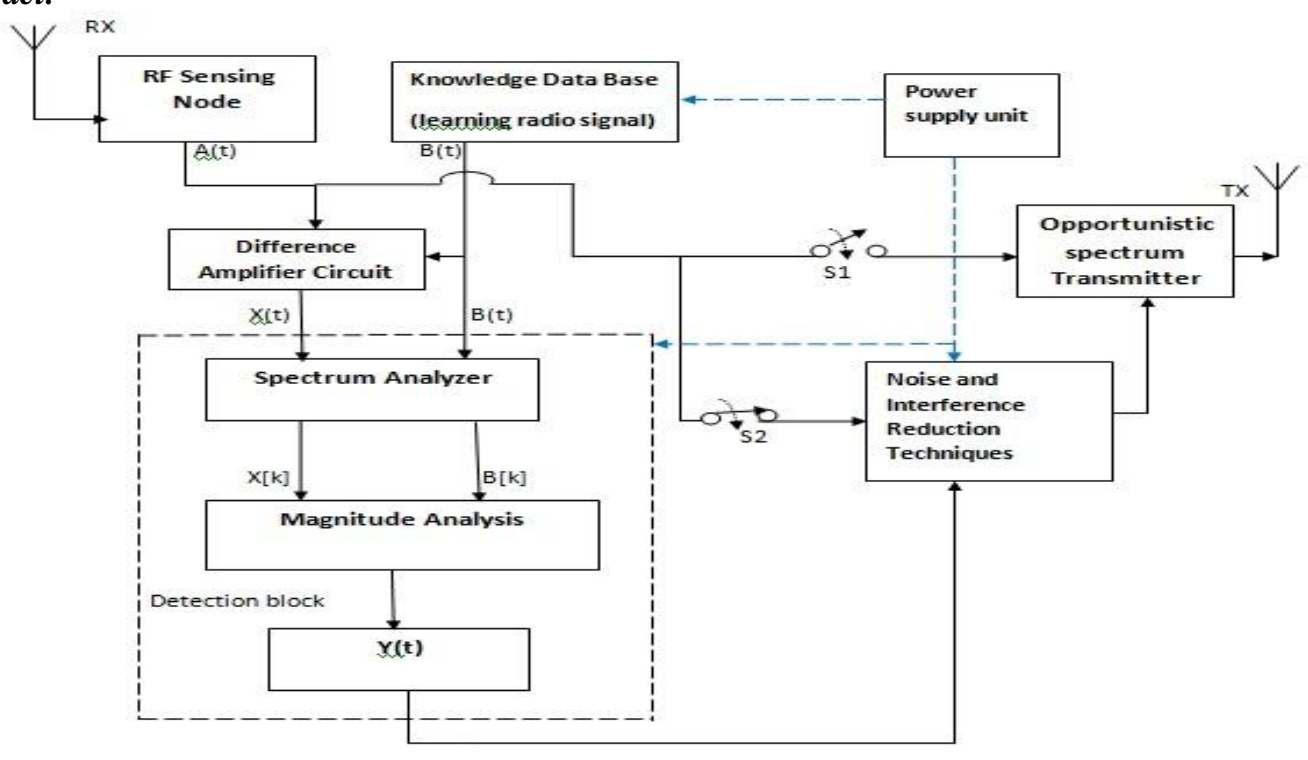

Figure-1 


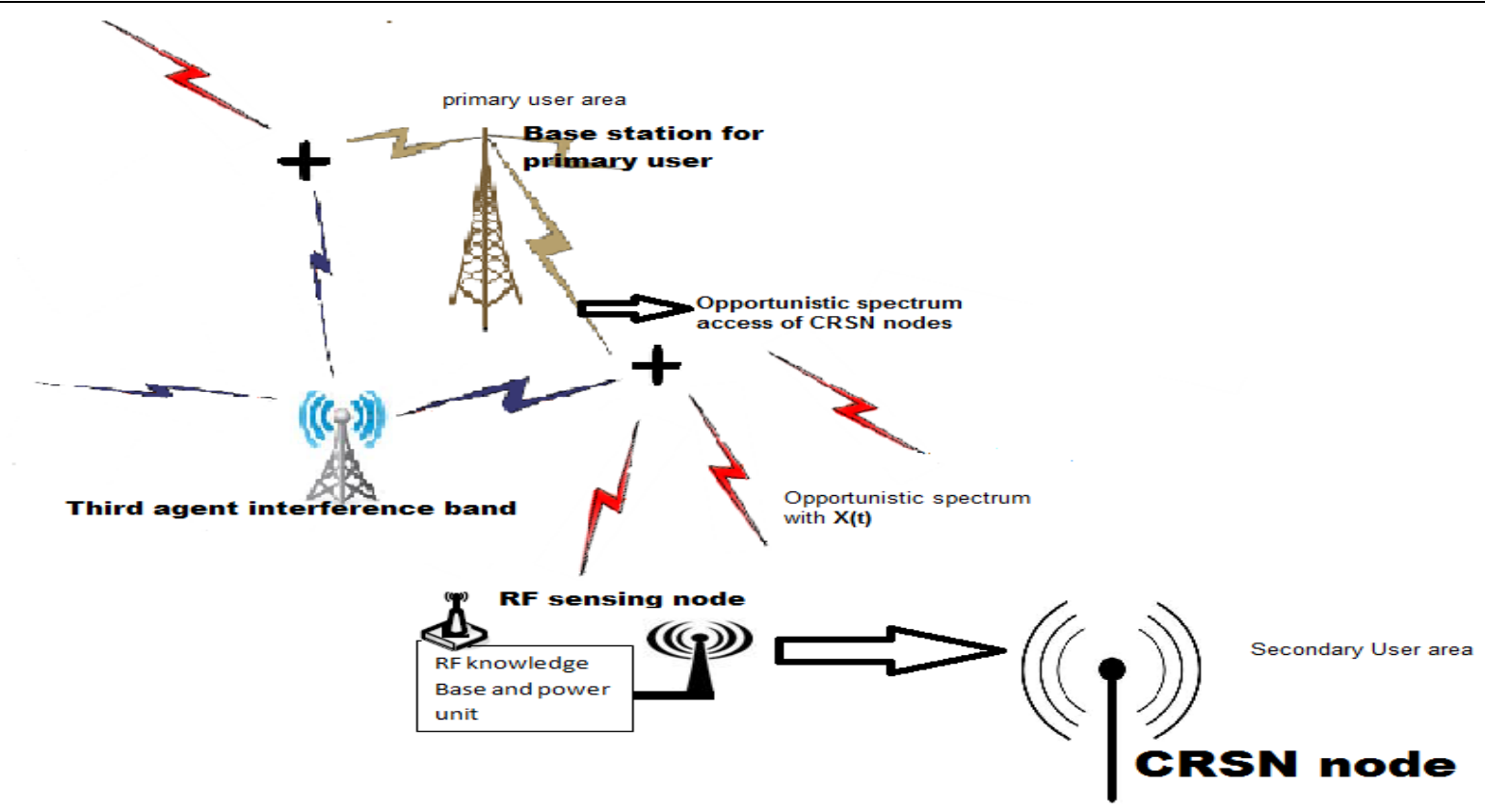

Figure-2

Figuer-1 and Figure-2 shows the problem in this cognitive radio where it can be rectified by prototype model. which will have ability in differentiate the signal is noise or interference. The prototype of the model represent the function of each block in RF knowledge base and power unit is the important aspect to be considered.

\section{i. Radio frequency sensing node:}

RF Test Solutions supplies RF Sensors component for a range of frequency is available in hardware prototype. Hardware consists of either dedicated high speed ( $\mathrm{GHz}$ per second sweep rate) receivers/scanners, or more generic Spectrum Analyzer based solutions. A number of pre-written software applications are available, or we can write your own as in[1].Applications include spectrum monitoring \& regulation for enforcement agencies, band owners, and regulators. Military and civilian users can use a RF Sensor Network with full DF capability to identify the location of a transmitter. Example for Mobile Phone/Wireless applications we also have available JDSU Scanner/Receiver hardware and software tools for coverage testing, interference finding \& signal analysis.

In these paper the radio frequency which differ in the range of magnitude or phase can be sensed by this RF sensing node as it access [3]. The RF signal which is sensed by the sensor is not recognized by the CRSN node due to the addition of third party signal to the Opportunistic spectrum band of the base station so the cognitive radio network can't access the Opportunistic spectrum.

The signal sensed is $\mathrm{A}(\mathrm{t})$, which is the sum of Opportunistic spectrum and $\mathrm{X}(\mathrm{t})$.

$$
A(t)=S(t)+X(t)
$$

Where, $\mathrm{S}(\mathrm{t})$ is Opportunistic spectrum available at the Base station and $\mathrm{X}(\mathrm{t})$ is interference or noise added . which may be equal in frequency and amplitude of $S(t)$.

\section{ii. Knowledge Data Base:}

By assuming the range of frequency and amplitude of the signal $s(t)$ is stored in the data base by learning algorithm manner. it gives the signal $\mathrm{B}(\mathrm{t})$ which is the part or sample of signal which is likely at the approximated range of $\mathrm{S}(\mathrm{t})$. these kind knowledge data base is done and accessed at [2].

\section{iii. A difference amplifier:}

A difference amplifier is a special purpose amplifier designed to measure differential signals, otherwise known as a subtractor. A key feature of a difference amplifier is its ability to remove unwanted common mode signals, known as common mode rejection (CMR). Unlike most types of amplifiers, difference amplifiers are typically able to measure voltages beyond the supply rails, and are used in applications where large dc or ac common-mode voltages are present. They are ideal for current and voltage monitoring. The simple circuit for the difference amplifier is given below figure-3 


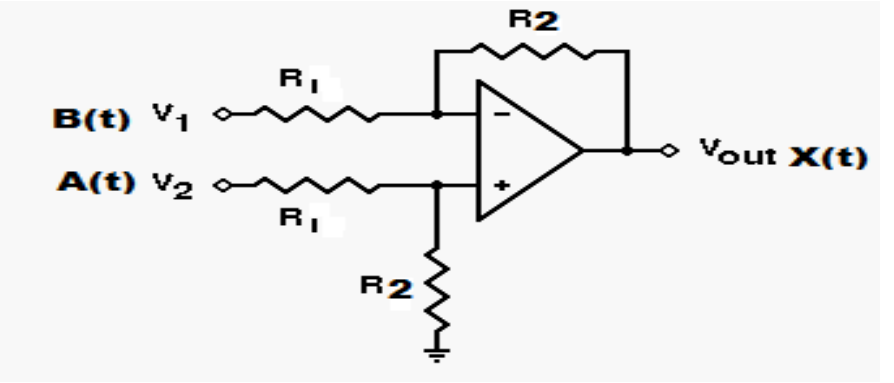

Figure-3

Here we took $\mathrm{B}(\mathrm{t})$ and $\mathrm{A}(\mathrm{t})$ as a two signal which is to be subtractred . the difference in the signal is taken as $\mathrm{X}(\mathrm{t})$.

Where the signal $X(t)=R 2 / R 1[A(t)-B(t)]$.

When $\mathrm{R} 1=\mathrm{R} 2$ then signal becomes

$$
X(t)=[A(t)-B(t)] .
$$

\section{SPECTRUM ANALYZER}

A spectrum analyzer measures the magnitude of an input signal versus frequency within the full frequency range of the instrument. The primary use is to measure the power of the spectrum of known and unknown signals. The input of spectrum analyzer is the signal $\mathrm{B}(\mathrm{t})$ and $\mathrm{X}(\mathrm{t})$. if the spectrum analyzer is dual channel then both the signals FFT are analyzed in [3]. The spectrum analyzer which gives the output spectrum range in time and frequency domain with same amplitude level is given in figure-4.

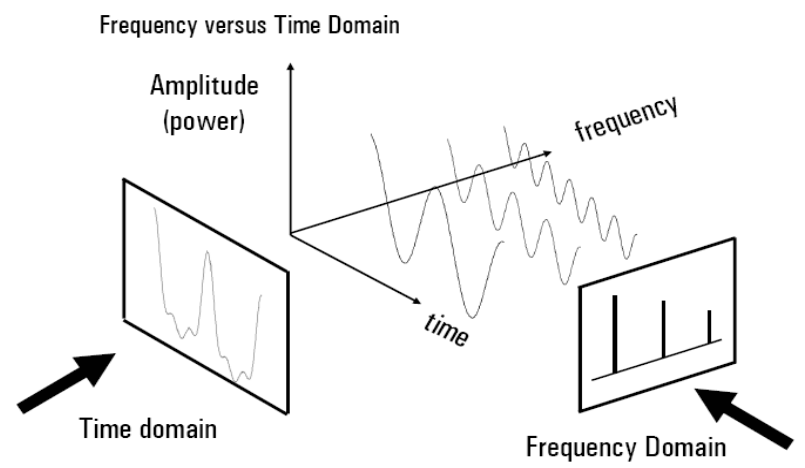

The FFT Spectrum Analyzer takes a time varying input signal, like you would see on an oscilloscope trace, and computes its frequency spectrum. Fourier's basic theorem states that any waveform in the time domain can be represented by the weighted sum of pure sine waves of all frequencies. If the signal in the time domain (as viewed on an oscilloscope) is periodic, then its spectrum is probably dominated by a single frequency component. What the spectrum analyzer does is represent the time domain signal by its component frequencies.The FFT of the signal is given by basic relationship of the discrete Fourier Transform:

$$
X[k]=\sum_{n=0}^{N-1} x[n] \exp (-i 2 \pi k n / N)=\sum_{n=0}^{N-1} x[n] W_{N}^{n k}
$$

Here the $\mathrm{X}[\mathrm{k}]$ is the Transform of $\mathrm{x}(\mathrm{t})$ and similarly for $\mathrm{B}[\mathrm{k}]$ is the Transform of $\mathrm{B}(\mathrm{t})$. the FFT Transformed signal is given below in figure-5.

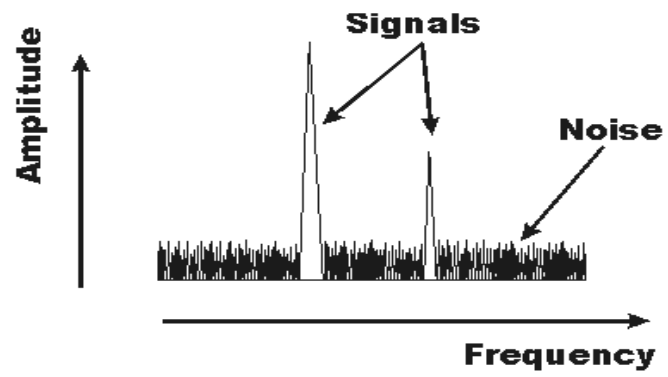

FTT of signal $\mathrm{X}(\mathrm{t})$ with noise and amplitude range. 
Finally the spectrum analyzer is use to give the amplitude range of FFT signal of each $\mathrm{X}[\mathrm{K}]$ and $\mathrm{B}[\mathrm{k}]$ signals which is used to analyses the magnitude and conclude the study by the performs of magnitude analyses.

\section{MAGNITUDE ANALYSIS}

Magnitude analysis compare the magnitude (amplitude) of the two spectrum $\mathrm{X}[\mathrm{k}]$ and $\mathrm{B}[\mathrm{k}]$ and confrim the spectrum $\mathrm{X}(\mathrm{t})$ is intereference or noise by the variable $\mathrm{Y}[\mathrm{t}]$. in the interference we have two types of interference.

1. Constructive interference

2. Destructive interference

The $\mathrm{Y}[\mathrm{t}]$ is given by the expression :

$$
\mathrm{Y}[\mathrm{t}]=\mathrm{B}[\mathrm{k}]+\mathrm{X}[\mathrm{k}] / \mathrm{B}[\mathrm{k}] .
$$

- If $\mathrm{Y}[\mathrm{t}]$ is greater than 1 then the signal which is added to $\mathrm{S}(\mathrm{t})$ (signal from the base station) is Constructive interference.

- Else if $\mathrm{Y}[\mathrm{t}]$ is lesser than 1 ie, negative value then the signal which is added to $\mathrm{S}(\mathrm{t})$ (signal from the base station) is Destructive interference.

- Else $\mathrm{Y}[\mathrm{t}]$ is varied from 0 to1 then the signal which is added to $\mathrm{S}(\mathrm{t})$ is noise.

$\mathrm{Y}[\mathrm{t}]$ block is use to analysis the signal is what type of process and enable the reduction block.

By the signal of $\mathrm{Y}[\mathrm{t}]$ values the reduction process varies in the form of:

1. Noise reduction Techniques.

2. Interference Techniques.

Noise and interference techniques can be achived by the varies method and each method has its own process and its limitation toward the reduction. As the noise can be achived by placing of approriate filters which is desinged for range of noise in the system.

\section{CON CLUSION}

In this paper we examined the problems associated with adversarial manipulation of cognitive radio sensory inputs, in an effort to cause the victim cognitive radio to behave suboptimally or maliciously. We showed how this ability to manipulate sensors introduces new threats beyond those typically associated with a wireless network.

\section{Reference :}

[1] "Calibration-Free RF-Based Localization Algorithm for Sensor Actuator Networks using Particle Filters" Aly I. El-Osery, Member, IEEE, Wael Abd-Almageed and Moustafa Youssef, IEEE, Members.

[2]. "Security in Cognitive Radio Networks:Threats and Mitigation". By T. Charles Clancy and Nathan goergen Electrical and Computer Engineering, University of Maryland, College Park.

[3]. "spectrum analyzer tenchiques" literature.agilent.com/litweb/pdf/5965-7920E.pdf.

[4]. "Cognitive Radio: Brain-Empowered Wireless Communications" Simon Haykin, Life Fellow, IEEE Journal On Selected Areas In Communications, Vol. 23, No. 2, February 2005. 